\title{
The Orbital Pointing Servo \\ A Miniature Pan/Tilt Mechanism
}

\author{
Pablo Bandera \\ Moog, Inc., Schaeffer Magnetics Division \\ 21339 Nordhoff St., Chatsworth, CA 91311 \\ phone: (818) 576-6804 fax: (818) 341-3884 \\ pbandera@sch.moog.com
}

\begin{abstract}
Moog, Inc., Schaeffer Magnetics Division, is currently developing a miniature pan/tilt mechanism called the Orbital Pointing Servo (OPS). This device is designed to point a small camera or sensor anywhere within a +/-45-degree optical cone at slew rates of up to 1100 degrees/second. Most pan/tilt mechanisms involve a motion-on-motion design, in which one motor controls one axis of motion while a second larger motor moves the first motor along with the payload in the other axis. The OPS is inherently more efficient in size, weight, speed, and power because the payload is mounted on a true ring-in-ring gimbal and controlled in both axes simultaneously by a single integrated mechanism.

While the OPS can be operated open loop, specially designed position sensors integrated directly into the gimbal itself provide position feedback for closed loop control. Aside from generally improving performance beyond the open loop characteristics, closed loop operation would allow the OPS to be used autonomously as part of a self-reliant vision system, or could reject vibrations and disturbances on a mobile robot to stabilize the image. The OPS could also be used to point a mirror for beam steering, small thrusters for thrust vector control, or various types of sensors.
\end{abstract}

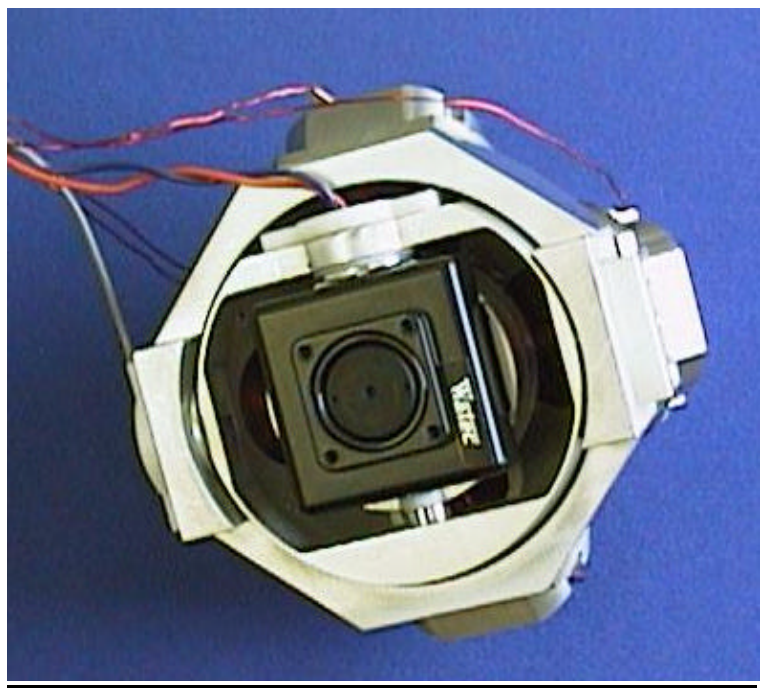

Figure 1. Orbital Pointing Servo with miniature camera

\section{The Need for Pointing Control}

Being able to visually survey the Martian surface proved to be of primary importance to the Pathfinder mission, where video images and photographs not only increased our understanding of Mars but helped to disseminate that information to the general public via the media. The popularity of the Mars missions planned for the next several years is largely due to people being able to literally see what Mars is like. None of this would be possible without an efficient and reliable vision system, and in the coming years mission requirements will only get more demanding. 
Ideally a visual image, whether it be of Mars, Earth, or the inside of a building, should have a high resolution to provide detailed information and a wide field of view to cover as much area as possible. Combining these two features, however, often results in a large amount of information being transferred back and forth, making it difficult to process the data quickly and causing telemetry or processing problems. A vision system involving a fixed wide-field and highresolution camera, therefore, is inherently slow and inefficient. A more practical system might involve a high-resolution camera with a narrower field of view that can be quickly and accurately pointed in any direction. A large area can then be surveyed by scanning over it without sacrificing resolution and without imposing unreasonable data processing requirements. The problem then becomes one of scanning speed and pointing accuracy. If the camera can move from one point to another in less time than it takes to process the data from each point, then no information is lost. If the pointing is accurate and repeatable, then the information is reliable.

While scanning and pointing are useful functions of a vision system, the ability to not move can be equally important. A camera mounted on a mobile robot, for example, should be able to maintain a steady focused image even while it is moving. A closed-loop pointing mechanism could accomplish this by actively rejecting vibrations and disturbances to stabilize the image during maneuvers. If this closed-loop functionality were to be integrated into the system control architecture, it could provide accurate tracking and navigation for the robot itself. Of course, in order for this to be feasible, the pointing mechanism must be extremely small and light, and for satellite applications it must consume very little power.

\section{Current State-of-the-Art}

To meet the demanding requirements of many current and future applications, therefore, a pointing mechanism must be very small, light, fast, and power-efficient. Most commercially available pointing or pan/tilt mechanisms, however, do not satisfy these requirements. The typical design involves a motion-onmotion configuration, in which one motor controls one axis while a second (usually larger) motor moves the first along with the payload about the orthogonal axis. These devices are inherently inefficient as only part of the work generated by the larger motor is being used to move the payload. These configurations also tend to be fairly large and heavy, and often involve some sort of gearing.

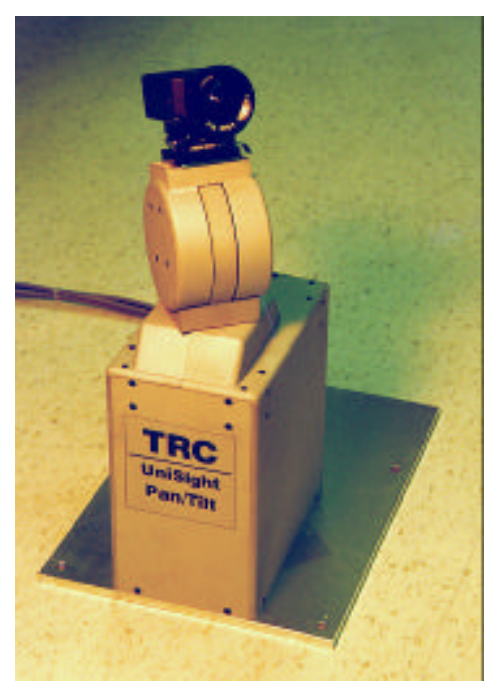

Figure 2. TRC Unisight mechanism

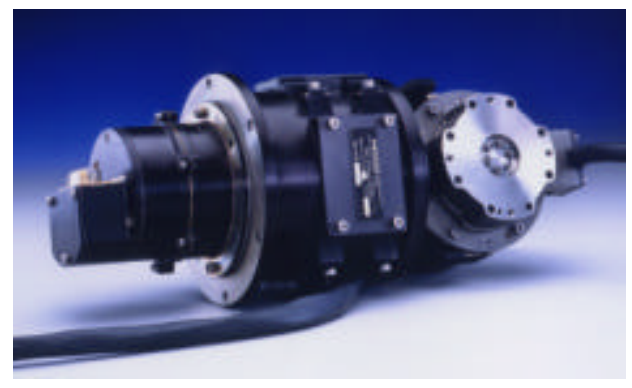

Figure 3. Moog SMD Type 11 Biax 
The TRC Unisight Pan/Tilt shown in figure 2, for example, is a popular pan/tilt mechanism for camera pointing. The camera, pan motor, tilt motor, and electronics are mounted vertically in a convenient package, and the range of motion is $+/-160$ degrees in pan and $+/-90$ degrees in tilt. However, the device consumes 400 Watts of power and weighs 6.4 $\mathrm{Kg}$. Because each axis is driven with a different motor, the maximum speed for each is different - $650 \mathrm{deg} / \mathrm{sec}$ in pan and 500 $\mathrm{deg} / \mathrm{sec}$ in tilt. Moog SMD itself has made pan/tilt mechanisms for space applications for the last 15 years, and is generally considered a leader in the industry. These, too, are motionon-motion designs, the smallest one being the type 11 gimbal shown in figure 3 . This device consumes only 5 Watts of power and can rotate 360 degrees in each axis. The maximum speed, however, is only $31 \mathrm{deg} / \mathrm{sec}$, and the total weight is $1.4 \mathrm{Kg}$

The ideal camera pointing design should not sacrifice size, weight, or speed for torque or range of motion. As can be seen from the specifications at he end of this paper, the Orbital Pointing Servo (OPS) will achieve the best balance of all these requirements. By controlling both axes simultaneously with a single integrated driving mechanism, the limitations and inefficiencies of a motion-on- motion design are eliminated. The result is a device that weighs 230 grams and is about the size of a baseball. The current prototype requires only $12 \mathrm{~W}$ of power to move 45 degrees, but the final design is expected to use $10 \mathrm{~W}$ or less. With no load on the armature, the OPS can reach slew rates in excess of $1000 \mathrm{deg} / \mathrm{sec}$. These numbers are based on a device which accommodates position sensors. For applications in which sensors are not needed, the performance is expected to improve significantly.

\section{OPS Description}

The Orbital Pointing Servo (OPS) shown in figures 1 and 4 is a miniature pan/tilt mechanism designed to point a small camera anywhere within a $+/-45$ degree range in any direction. Unlike other pan/tilt designs which use the principle of motion-on-motion to achieve two degrees of freedom, the OPS has only one moving part mounted on a true gimbal and driven by a single two-dimensional motor. This allows the device to be much smaller and lighter and is inherently more efficient. There is currently a prototype being tested that weighs only 230 grams and measures $6 \mathrm{~cm}$ by $6 \mathrm{~cm}$ by $4 \mathrm{~cm}$. Initial tests show that about 12 Watts of power are required to move 45 degrees in pan or tilt.

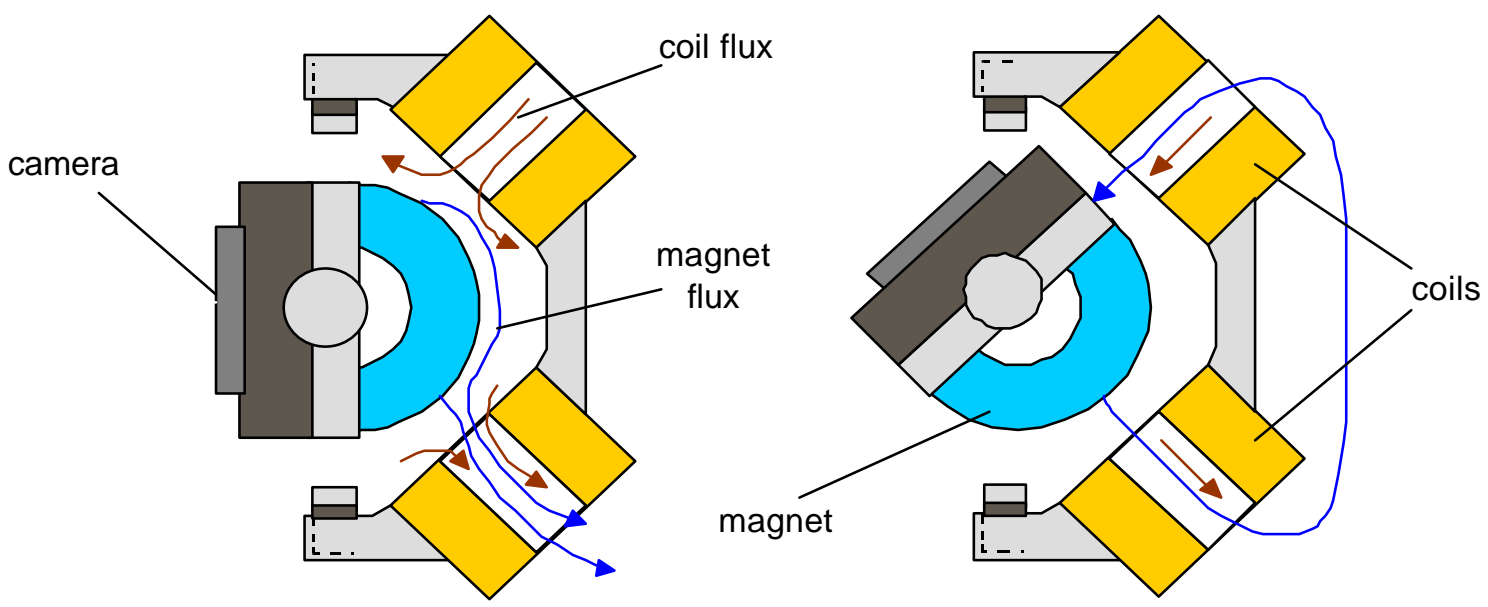

Figure 4. Cross-section of OPS showing basic operation along one axis 
The camera itself is a miniature video camera such as the Watek CCD currently used on the prototype or JPL's Active Pixel Camera. This is mounted onto the OPS armature, which is made up of a disc-shaped camera holder and a hemispherically shaped permanent magnet. This in turn is mounted on a true ring-in-ring gimbal in such a way as to make the armature's center of mass coincide with the center of rotation. This results in a minimuminertia mass-balanced design with symmetrical operation in any direction about a single point of rotation.

The driving element of the OPS is a fixed configuration of four coils mounted orthogonally on the sides of a four-sided pyramid structure. These coils effectively surround the curved face of the hemispherical magnet, with their centerlines pointing toward the center of rotation. Applying current to the coils generates a magnetic field around the magnet. Because magnetic fields sum vectorally, the resultant total field causes the magnet to align itself to a new equilibrium position (refer to figure 4). By varying the ratio of currents in the coils, the magnet can be positioned anywhere within a $+/-45$ degree optical cone. The camera, mounted on the flat face of the magnet, looks out the open bottom of the pyramid structure, which offers a wide and unobstructed field of view.

While the OPS can be operated open loop, specially designed sensors can provide position feedback for closed loop control. To keep the size and weight of the total mechanism down, a novel design was created that integrates the sensors with the gimbal itself. The shafts that support the gimbal rings pass through radial retainer bearings and rigidly connect to the moving member of the position sensor. This member, which rotates relative to the rest of the sensor, is capacitively coupled to two plates, marked " $A$ " and " $B$ " in figure 5. Together these parts
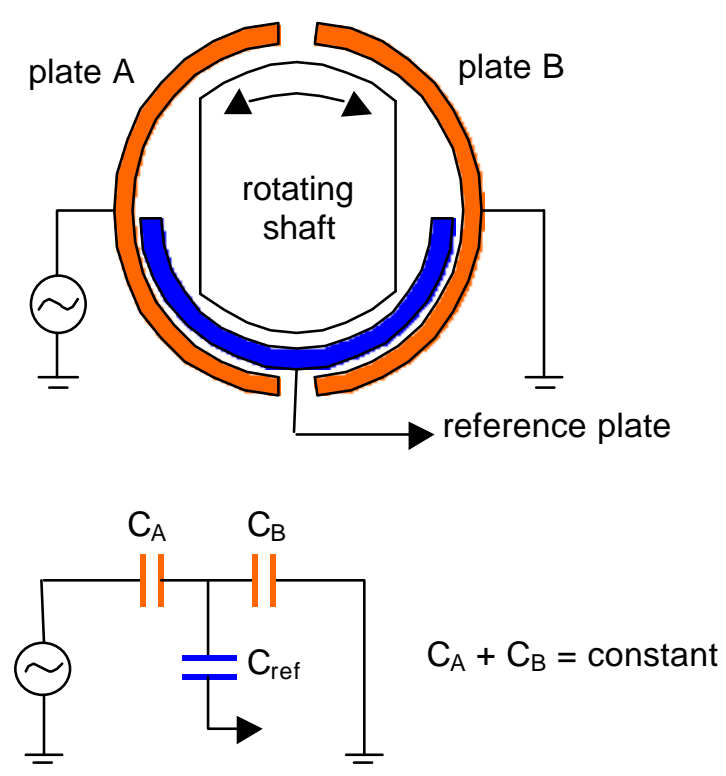

Figure 5. OPS position sensor design and equivalent circuit

effectively act as an AC voltage divider. The amplitude of the AC voltage sensed on the moving plate is a function of the relative overlap with plates $A$ and B. In order to eliminate the problem of flexing wires which would decrease the life and reliability of the sensor, a stationary "reference" plate is AC coupled to the moving plate over the entire range of motion. In this way all the wiring can be rigidly connected to fixed parts, and the sensor will not produce any friction or moments on the gimbal at all.

The OPS was originally designed in cooperation with Comptek Amherst Systems in Buffalo, N.Y. to be used with their machine vision technology. Comptek's dynamic reconfigurable camera is based on CMOS Active Pixel Sensor (APS) technology being developed at JPL. Among the various advantages over other imagers, a CMOS APS camera has the capability to overlap windows, thus providing for a wide field-of-view background window at lower resolution overlapped by up to two smaller highresolution "targeting" windows (refer to figure 


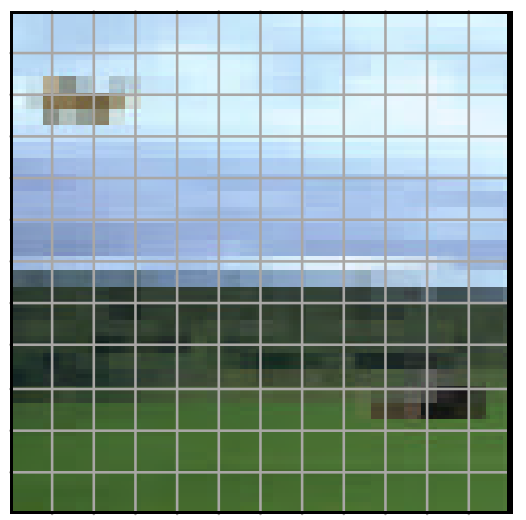

$\mathbf{t}=\mathbf{1}$

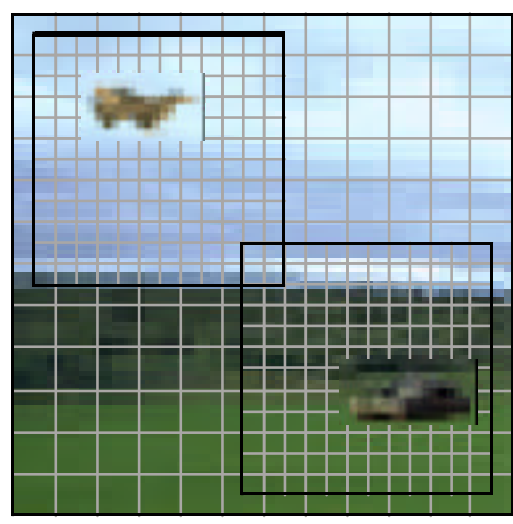

$\mathbf{t}=\mathbf{2}$

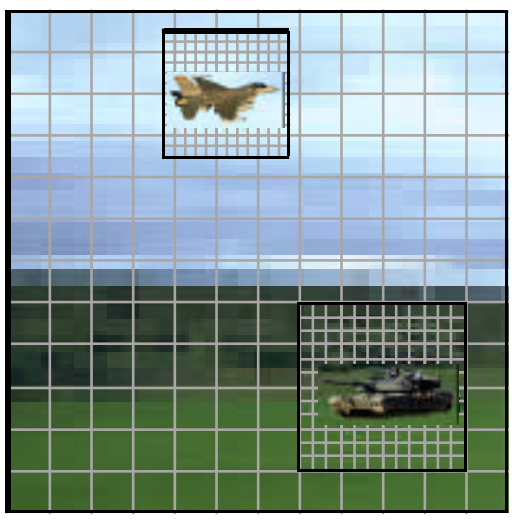

$\mathbf{t}=\mathbf{3}$

Figure 6. Example of APS non-destructive pixel technology, with two high-resolution windows overlapping a full-field-of-view lower-resolution window.

6). This feature allows the system to simultaneously view two targets at high resolution, maintain total situation awareness with a lower-resolution background window, and still have fast (hundreds of $\mathrm{Hz}$ ) frame rates due to the intelligent allocation of video resources. Combining this with the pointing capability of the OPS results in an extremely versatile and high-performance tracking and imaging system for a number of different applications.

\section{$\underline{\text { OPS System Integration }}$}

Probably the most attractive feature of the OPS is the ease with which it can be accommodated physically into a system or payload. Its hemispherical shape and small dimensions allow the OPS to fit into a very small envelope or even in between other components. It can be mounted from the back, behind the camera, or from the sides. Because the gimbaled camera is contained within the pyramid structure, there is no need to provide any clearance for a moving mechanism, as would be the case with any motion-on-motion device. The entire outer structure is fixed and can be so enclosed by the surrounding system as to be physically touching on all sides except the front.
All the wiring for the coils, sensors, and camera are routed out the back. The driver, controller, and sensor electronics can be mounted on the back or located remotely, depending on the desired system architecture. Because there is only one moving part and no gearing or transmission, mechanical debris is not an issue. There is, however, a significant magnetic field which could cause electromagnetic interference with neighboring systems. The Gauss levels around the back and sides of the device immediately surrounding the coils may be on the order of 100 Gauss for a design similar to that of the current prototype. It may be necessary to use a magnetic steel circuit to contain and guide the flux, in which case the Gauss levels would be at least two orders of magnitude less. The field in front of the camera should be considerably less as all the electromagnetic components act on the back side.

The electronic requirements are not well known at this time, as future developments to the OPS design will in large part determine the required power and control algorithm. As a minimum, two independent channels with two current drivers will be needed. For a closed loop design, the same is true for the control circuitry. 


\section{OPS Prototype Performance}

\section{Static Performance}

Open loop tests have been conducted using a four-way quiescent control algorithm. This scheme keeps the total current to each pair of coils constant (i.e. the total current controlling each axis is constant). In this way, the angular travel of the armature is a function of the quiescent coefficient $c$ according to the following equations...

$$
\begin{aligned}
& I_{1}=c \frac{I_{\text {total }}}{2} \\
& I_{2}=(1-c) \frac{I_{\text {total }}}{2}
\end{aligned}
$$
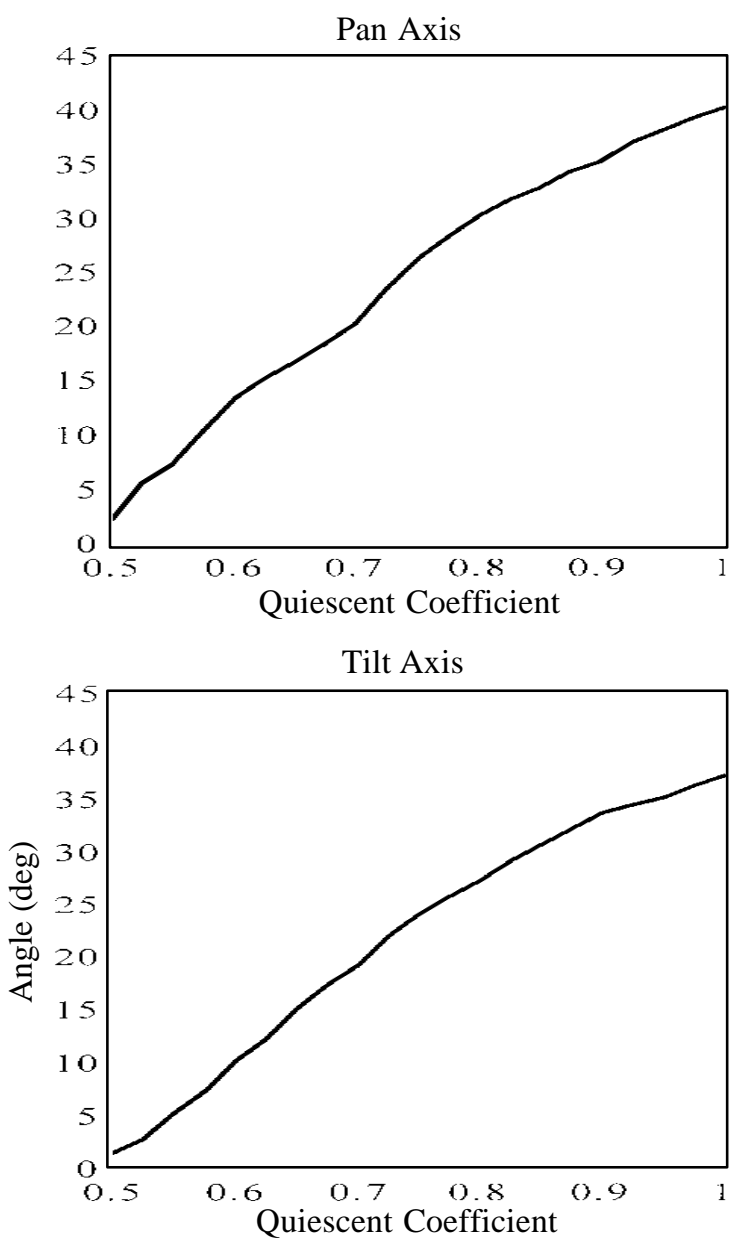

Figure 7. Position Gain of OPS Prototype
$I_{1}$ and $I_{2}$ are the currents driving each coil in an axis-pair and $I_{\text {total }}$ is the total quiescent current applied to the OPS (each axis receives half this value). With $c$ varying between 0 and 1 for either pan or tilt, the armature should move between $-45^{\circ}$ and $+45^{\circ}$. The open-loop controller linearly maps the input position commands to $c$.

The data in figure 7 was taken with one axispair active while the other axis-pair was turned off. A total quiescent current of three Amps was used. Ideally, with no external forces acting on the armature, it should reach 45 degrees for $c=1$. However, since the center of mass of the armature on this prototype is located behind the point of rotation, there is a constant centering torque due to gravity, which prevents achieving the full range of travel. This problem will be eliminated by simply balancing the armature in the gimbal so that its center of mass coincides with the point of rotation.

The fact that the pan axis travels about 3 degrees more than the tilt axis is due to the fact that the latter must move the inner gimbal ring along with the payload, while the former does not. Again, this asymmetry will be taken care of when the armature is balanced.

An important thing to keep in mind when discussing performance is that this prototype was designed to accommodate position sensors for future closed-loop testing (the results presented in this paper are of open-loop performance). If sensors are not needed, the mechanical design of the OPS can be much more efficient, which in turn results in a more efficient magnetic design. Consequently, an open-loop OPS, designed as such, will produce significantly more torque and consume less power than the prototype currently being studied. This will be investigated in more detail in the near future. 


\section{Dynamic Performance}

A number of step response tests were performed with various input amplitudes and quiescent currents. Typical results are shown in figure 8. Once again, a slight difference in performance can be seen between the pan and tilt axes due to the imbalance of the armature in this prototype. The added mass of the gimbal ring in the tilt axis causes the response to be somewhat slower than that of the pan axis. In the pan axis, the armature has a rise time of 69.3 milliseconds, a settling time of 159 milliseconds, and a maximum angular velocity of $587 \mathrm{deg} / \mathrm{sec}$. In the tilt axis, the armature has a rise time of 93.7 milliseconds, a settling time of 191 milliseconds, and a maximum angular velocity of $530 \mathrm{deg} / \mathrm{sec}$. For a 50\% larger input signal, the maximum velocity of the pan axis was measured at 1090 $\mathrm{deg} / \mathrm{sec}$.
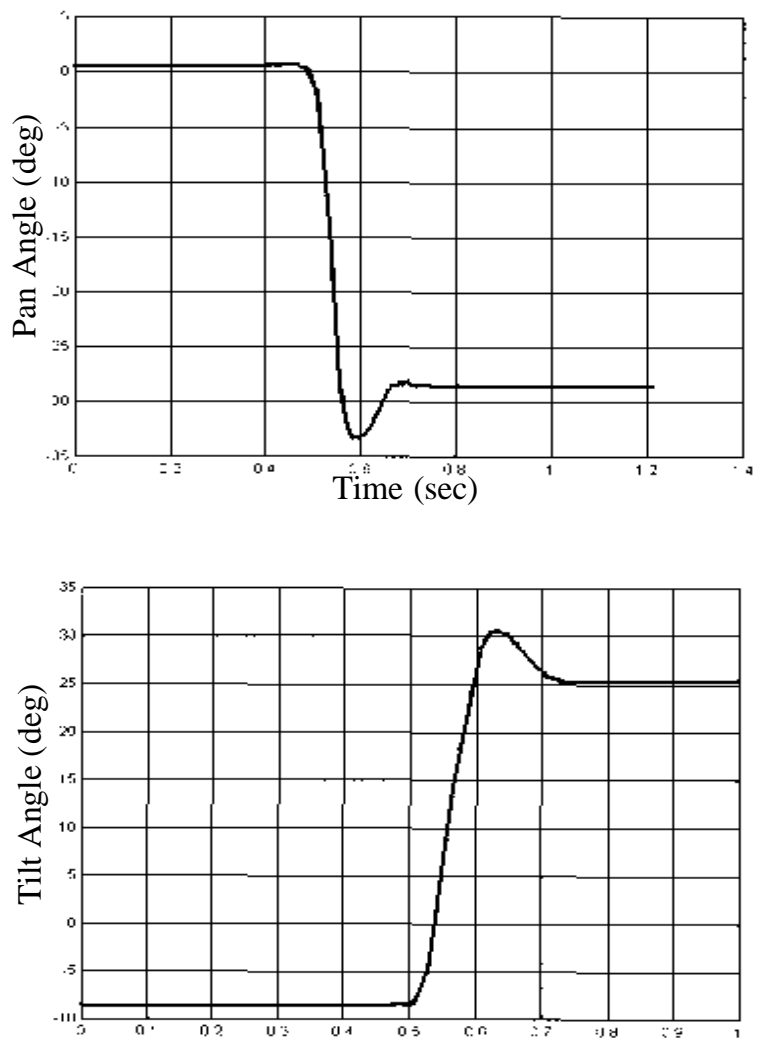

Figure 8. Step Response of OPS Prototype

\section{Conclusion}

In an increasingly demanding market where size, weight, and power must be minimized, the OPS promises to be a significant improvement over current pan/tilt mechanisms for many applications. By maintaining a 90degree field of regard in a compact and robust package, the OPS lends itself to applications ranging from ground-based military vehicles to robotic systems to small satellites. The current prototype not only proves the concept of a miniature, integrated pan/tilt mechanism, but potentially opens up pointing, scanning, and tracking capabilities for small vehicles that may not have existed before.

There are a number of hurdles to get over before the development of the OPS can be considered complete. The easiest problem to solve is that of balancing the armature to achieve more symmetrical performance in both axes of motion. A more significant goal is closing the loop around position with the integrated sensors. This will improve the dynamic performance of the OPS significantly and open up many more potential applications in areas involving such functions as tracking, navigation, and autonomous control. The improved performance of an open-loop OPS will also be characterized in the near future. 